\title{
Broadband Millimeter-Wave Textile-Based Flexible Rectenna for Wearable Energy Harvesting
}

\author{
Mahmoud Wagih, Student Member, IEEE, Geoffrey S. Hilton, Alex S. Weddell, Member, IEEE, \\ and Steve Beeby, Senior Member, IEEE
}

\begin{abstract}
Millimeter-Wave (mmWave) bands, a key part of future 5G networks, represent a potential channel for RF energy harvesting, where the high-gain antenna arrays offer improved end-to-end efficiency compared to sub-6 GHz networks. This paper presents a broadband mmWave rectenna, the first rectenna realized on a flexible textile substrate for wearable applications. The proposed novel antenna's bandwidth extends from 23 to $40 \mathrm{GHz}$, with a minimum radiation efficiency of $67 \%$ up to 30 GHz, over $3 \mathrm{~dB}$ improvement compared to a standard patch. A stable gain of more than $8 \mathrm{~dB}$ is achieved based on a textile reflector plane. The antenna is directly connected to a textilebased microstrip voltage doubler rectifier utilizing commercial Schottky diodes. The rectifier is matched to the antenna using a tapered line feed for high-impedance matching, achieving broadband high voltage-sensitivity. The rectifier has a peak RFDC efficiency of $12 \%$ and a $9.5 \mathrm{dBm} 1 \mathrm{~V}$ sensitivity from 23 to 24.25 GHz. The integrated rectenna is demonstrated with more than 1.3-V DC output from $12 \mathrm{dBm}$ of $\mathrm{mm}$ Wave wireless power across a $28 \%$ fractional bandwidth from 20 to $26.5 \mathrm{GHz}$, a $15 \%$ half-power fractional bandwidth, and a peak output of $6.5 \mathrm{~V}$ from $20 \mathrm{dBm}$ at $24 \mathrm{GHz}$.
\end{abstract}

Index Terms-Antennas, Broadband matching networks, Internet of Things, Microstrip antennas, Rectennas, Rectifier, RF Energy Harvesting, Wearable Antenna, Wireless Power Transfer

\section{INTRODUCTION}

$\mathbf{M}$ ILLIMETER-WAVE (mmWave) bands will play a significant role in future $5 \mathrm{G}$ networks, where the broad spectrum aims to mitigate traffic-related issues in existing sub-6 $\mathrm{GHz}$ networks. Although mmWaves suffer from increased propagation losses due to absorption by air and water molecules around 20 and $60 \mathrm{GHz}$, respectively, the large number of channels, beamforming and high-gain large antenna arrays are attractive features of mmWave networks [1]. The low-cost, pervasive Internet of Things (IoT) market not only benefits from the wider spectrum allowing trillions of devices to communicate, but also from the potential of utilizing

This paper is an expanded version from the IEEE Wireless Power Week, London, UK, June 17-20, 2019. This work was supported by the European Commission through the project EnABLES: European Infrastructure Powering the Internet of Things, funded under H2020-EU.1.4.1.2. Datasets in this paper are available from the University of Southampton repository at DOI: 10.5258/SOTON/D1493 (Corresponding author: Mahmoud Wagih)

M. Wagih, A. S. Weddell and S. Beeby are with the School of Electronics and Computer science, University of Southampton, Southampton, SO17 1BJ, U.K. (email: \{mahm1g15, asw, spb\} @ecs.soton.ac.uk)

G. S. Hilton is with the Department of Electrical and Electronic Engineering, University of Bristol, Bristol BS8 1UB, U.K. (email: Geoff.Hilton@bristol.ac.uk)

Digital Object Identifier:
mmWave bands to achieve power-autonomy through wireless energy harvesting.

Wireless Power Transfer (WPT) and Radio Frequency Energy Harvesting (RFEH) networks with Simultaneous Wireless Information and Power Transfer (SWIPT) at mmWave bands are expected to co-exist in future 5G networks [2], where a key research challenge is enabling wireless powering of Internet of Everything (IoE) networks as a fundamental component of $5 \mathrm{G}$ networks [3]. RFEH in a $28 \mathrm{GHz}$ network has been investigated numerically demonstrating improved energy and information coverage over sub-6 GHz networks, promising improved end-to-end RFEH efficiency over existing cellular networks [4], [5]. The improvement in energy-coverage and hence end-to-end efficiency can be attributed to the beamforming techniques based on base-stations with large antenna arrays which can be realized in smaller areas, due to the antennas' reduced size. Moreover, area constrained applications such as single-chip active RFID transmitters benefit from mmWave WPT due to the higher achievable antenna efficiencies with sub-mm ${ }^{2}$ aperture size [6].

Rectennas for WPT at mmWave frequencies have been previously presented, with focus on the 24 [7], [8] and 60 $\mathrm{GHz}$ license-free spectrum [9]. A circularly-polarized cavitybacked rectenna array [7], a Substrate-Integrated Waveguide (SIW) rectenna [10], as well as inkjet printed flexible rectennas on paper [11] and high-frequency Liquid Crystal Polymer (LCP) [12] have been reported. However, the rectennas have mostly been studied under single-tone excitation at $24 \mathrm{GHz}$. SIW structures have been used in mmWave rectennas due to their ability to improve the antennas' gain and isolation from surroundings, nevertheless, a SIW rectenna would normally target single bands and require an external microstrip matching network along with a SIW to microstrip transition, adding to the system's complexity [10]. Finally, while various antenna designs and matching architectures have been reported sub$6 \mathrm{GHz}$ [13], mmWave rectennas have been mostly limited to patch arrays and SIW antennas.

In order to overcome the cost-barrier of mmWave rectennas fabricated on low-loss RF substrates, additive manufacturing has been used to realize mmWave rectennas for pervasive IoT [12], [14], [15]. While the reported additively manufactured rectennas achieve a $1-\mathrm{V}$ output from as low as $10 \mathrm{dBm}$ of mmWave power, they were all studied under single-tone excitation and are expected to have their bandwidth bottlenecked due to the use of patch antennas and narrow-band beamforming or array-feed networks. Therefore, their ability to be extended to harvest power from the full $5 \mathrm{G}$ commercial 
spectrum is limited.

Although most low power mmWave rectennas are only able to yield a net DC power of less than $10 \mathrm{~mW}$, the justification for development of mmWave rectennas despite their lower efficiency compared to Ultra-high Frequency (UHF) bands lies in their compactness [6]. Moreover, mmWave transceivers have been reported with as low as $7.2-\mu \mathrm{W}$ active receiver power consumption [16], in addition to $\mathrm{nW}$-sleep power for Bluetooth Low Energy transceiver [17]. Furthermore, a low data rate $60 \mathrm{GHz}$ transceiver has been reported with a $4.9 \mathrm{pJ} / \mathrm{bit}$ energy consumption at $60 \mathrm{GHz}$ [18]. Therefore, developing an unobtrusive wearable mmWave rectenna, with a net DC output across a broad bandwidth using low-cost materials and commercial components is paramount for enabling mmWave RFEH.

In this paper, rectenna development for 5G RFEH and WPT is presented. A broadband textile antenna is presented for wearable applications, the first textile-based antenna covering the $5 \mathrm{G}$ spectrum. The rectenna is integrated with a fullydistributed microstrip voltage doubler rectifier based on a commercial Schottky diode demonstrating a 1.3-V DC output from less than $12 \mathrm{dBm}$ of mmWave power across the full bandwidth between 20 and $26.5 \mathrm{GHz}$, and more than $1-\mathrm{V}$ from under $10 \mathrm{dBm}$ from 23 to $24.5 \mathrm{GHz}$.

In addition to the contributions in [19], this paper presents substantial extensions in improving original broadband highefficiency antenna design, for more stable radiation patterns, efficiency and gain, independent of separation from the human body, in sections II and III. Additional analysis of the proposed antenna and comparisons to a standard patch antenna are presented.

The main novel contribution is the design and implementation of a broadband mmWave rectifier using a fully-distributed elements microstrip matching network, for compatibility with a standard $50 \Omega$ antenna, presented in section IV. This work demonstrates the first textile-based, flexible mmWave rectenna for wearable RFEH applications in future $5 \mathrm{G}$ mmWave bands (section $\mathrm{V}$ ), and the first rectenna with broadband $(28 \%$ fractional bandwidth) $1.3 \mathrm{~V} \mathrm{DC}$ sensitivity from $12 \mathrm{dBm}$ of wireless power above $20 \mathrm{GHz}$.

\section{ANTENNA DESIGN AND FABRICATION}

\section{A. High-Efficiency, Broadband Antenna Design}

$5 \mathrm{G}$ mmWave bands are motivated by the wider available spectrum. In Europe, China, Africa and Brazil, $26 \mathrm{GHz}$ is chosen as a center frequency while in the US, Japan, South Korea and India the legislated frequency is $28 \mathrm{GHz}$ [20]. In addition, $1 \mathrm{GHz}$ bandwidth will be allocated to individual mobile service providers in the so-called mmWave 5G bands [20]. Collectively, the 26 and $28 \mathrm{GHz}$ bands cover a bandwidth of $5.25 \mathrm{GHz}$, approximately $20 \%$ fractional bandwidth. Such wide bandwidth motivates research into broadband antenna design both for communication and wireless power harvesting applications.

In order to realize a rectenna capable of harvesting energy at $5 \mathrm{G}$ bands, along with the $24 \mathrm{GHz}$ license-free band, the antenna needs to have a wide impedance bandwidth. Moreover,

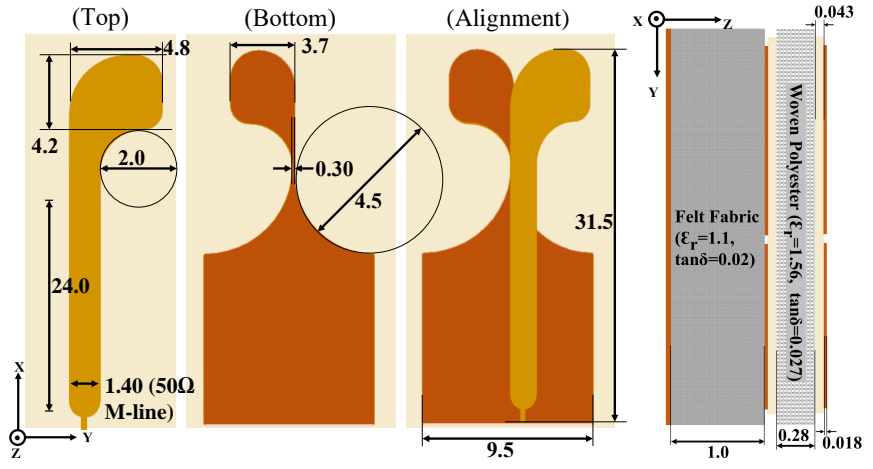

Fig. 1. The proposed antenna layout and dimensions (in $\mathrm{mm}$ ), showing the double-sided AVA and the substrate composition

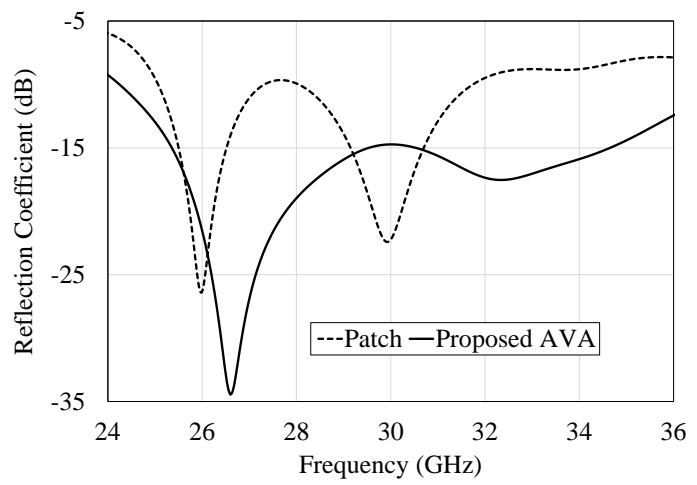

Fig. 2. Simulated reflection coefficient of the two antenna designs considered, a common-geometry patch and the proposed novel AVA.

as a textile or flexible rectenna may be used in proximity with human tissue, a frequency-independent antenna is preferred to minimize any detuning impact. The proposed antenna is based on the broadband Antipodal Vivaldi Antenna (AVA) [21]. Although the antenna's geometry is not self-complimentary or symmetric due the $50 \Omega$ microstrip line width being comparable to the radiator, resulting in a narrower bandwidth, the radiator's shape and size have been tuned in 3D full-wave simulation to achieve a bandwidth covering the 24,28 and 36 $\mathrm{GHz}$ bands.

The main aim of the antenna design process has been to prioritize maximizing the radiation efficiency. Given the high $\tan \delta$ of textile substrates [22], this has been achieved by choosing an antenna design where a minimum part of the radiator is parallel to the ground-plane (e.g. a monopole) to minimize the electric field dissipation in the substrate. This is explored in comparison to a standard patch antenna in Section II-B. The AVA's layout and dimensions are shown in Fig. 1.

\section{B. Comparison with a Standard Patch Antenna}

For benchmarking purpose, a standard microstrip patch antenna has been simulated to validate the assumptions on the influence of the radiator's area on the antenna's efficiency, and to demonstrate the improvement of the proposed design over a standard design on the same substrate. Patch antennas have been widely reported in wearable mmWave antennas [23] and in low-cost additively manufactured rectennas [12], [14], 


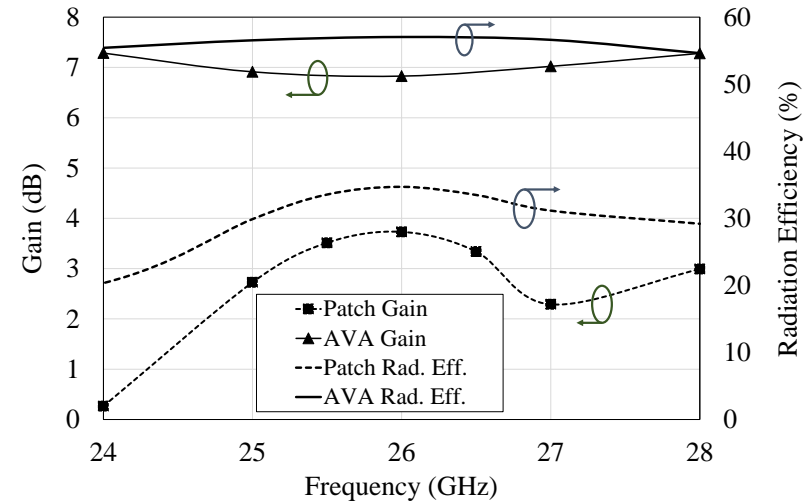

Fig. 3. Simulated gain and radiation efficiency of the patch antenna and the proposed AVA at the frequencies of interest.

[15]. The patch's dimensions are $9 \times 7.125 \mathrm{~mm}$ with a 3.25 $\mathrm{mm}$ inset microstrip feed. Both antennas have been simulated using CST Microwave Studio using the measured substrate properties $\left(\epsilon_{r}=1.67\right.$ and $\left.\tan \delta=0.027\right)$. Fig. 2 shows the simulated reflection coefficient of the antennas. The gain and radiation efficiency are shown in Fig. 3.

Overall, a more broadband behavior is expected from the proposed AVA, as demonstrated by the lower reflection coefficient in Fig. 2 and more stable gain in Fig. 3. However, at $26 \mathrm{GHz}$, where both antennas achieve $\mathrm{S}_{11}<-20 \mathrm{~dB}$, the proposed AVA achieves over $67 \%$ higher radiation efficiency relative to the patch antenna. This in-turn affects the antenna's gain demonstrating more than $3 \mathrm{~dB}$ gain improvement by the proposed antenna design over a common geometry patch. This is explained by the patch antenna's higher dielectric losses discussed earlier; over $53.7 \%$ of the accepted power is dissipated in the patch's textile substrate as opposed to only $32.4 \%$ of the power accepted by the proposed AVA.

\section{On-Body Antenna Isolation}

Obtaining stable off-body broadside radiation patterns is essential for efficient wearable rectenna operation. In [19], a novel approach of employing the human body as a reflector has been devised to improve the antenna's efficiency, bandwidth, and gain at the frequencies of interest. This has been achieved using asymmetric alignment of the radiator arms to allow the far-fields to reflect off the human body, hence improving the gain. However, this approach is only valid when the antenna is separated from the human body by more than $4 \mathrm{~mm}$, which cannot be guaranteed in all modes of operation.

In order to ensure the antenna maintains stable radiation patterns and bandwidth in the presence and absence of the human body, an additional metal reflector layer is proposed. Thus, broadside radiation patterns can be achieved by the monopole at its fundamental frequency. Metalized microwave shielding fabric (copper and nickel coated) has been used for the reflector plane. Shielding fabrics are chosen as they are breathable, more user-friendly compared to copper sheets and, in this case, do not need to be patterned to produce small features.

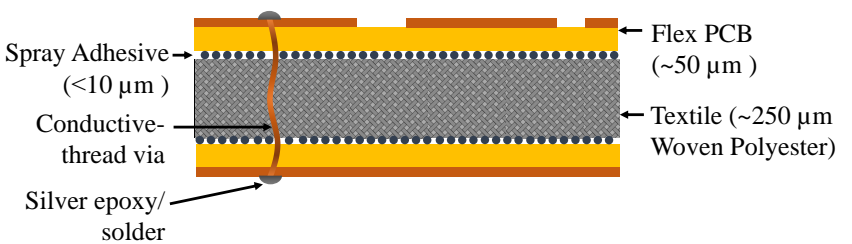

Fig. 4. Cross-section of the antenna showing the multi-layer substrate and the through-textile via.

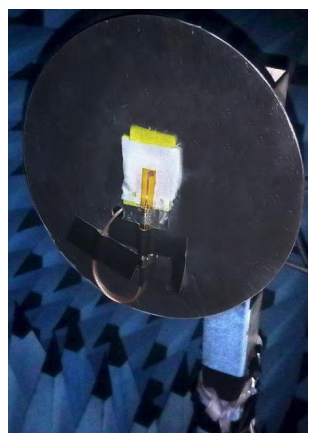

(a)

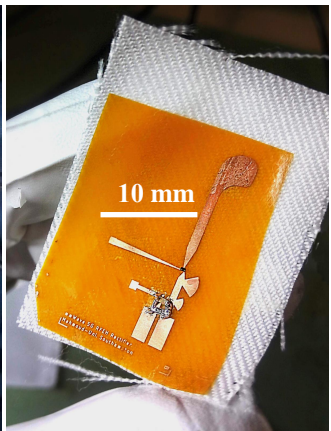

(b)

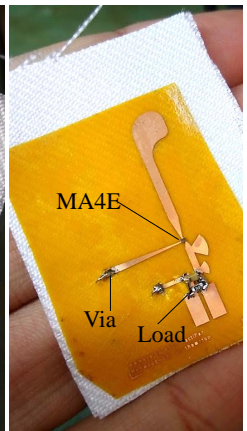

(c)
Fig. 5. Photographs of: (a) the connectorized antenna used for $S_{11}$ and radiation properties measurements, (b) the rectenna after mounting components before adding the through-textile via, (c) the rectenna used in the wireless testing.

The minimum separation between the antenna and the reflector has been set to $1 \mathrm{~mm}\left(0.08 \times \lambda_{0} /\right.$ at $\left.24 \mathrm{GHz}\right)$. A 1 $\mathrm{mm}$ felt fabric (of lower $\tan \delta$ compared to woven polyester) has been inserted between the antenna and the reflector as shown in Fig. 1 to support the reflector. While the additional fabric layer will degrade the antenna's radiation efficiency (due to the additional dielectric losses), the effect is expected to be minimal compared to degradation in the efficiency due to absorption by the human body.

\section{Antenna Fabrication}

Among the candidates for the antenna's fabrication methods are screen and inkjet-printing [24], woven or adhered conductive textiles and copper foils [22], [23], as well as etched Kapton copper laminates [25]-[27]. To produce smooth and homogeneous conductors, a high resolution process is crucial at mmWave bands, where the feature size is commonly less than $1 \mathrm{~mm}$. Therefore, manufacturing tolerances, surface roughness and non-uniformities need to be minimized as they can significantly affect the antenna's performance. As textile substrates are porous and have high surface roughness, conductor printing has been excluded due to the high thickness of the required interface layer, resulting in higher dielectric losses and reduced antenna efficiency [22].

Ultra-thin polyimide copper laminates exhibit improved flexibility compared to copper foils, and lower thickness compared to printed interface layers, minimizing dielectric losses. The antenna is fabricated on the polyimide laminates using a standard photolithography process, with the track's pattern plotted on a dark-film mask. A minimum feature size of $150 \mu \mathrm{m}$ is achieved using standard PCB manufacturing 
equipment. After the adhesion of the flexible circuit onto the textile substrate, the via holes are drilled and conductive thread is inserted to form the conductor. The antenna has been fabricated using the standard photolithography process described in [19], [26]. However, to realize the double-sided voltage doubler rectifier, conductive threads are utilized to create DC vias, solder paste has been used to connect the via (thread) to the copper traces on the top and bottom layers. Fig. 4 shows a cross-section of the structure. The fabricated antenna and rectenna prototypes are shown in Fig. 5.

While the fabrication processes reported in [23] and [28] can produce antenna's with re-producable $0.2 \mathrm{~mm}$-sized features, they utilize copper foils and embroidered conductive threads, respectively. The proposed method utilizing polyimide-copper laminates allows over-all thinner and lighter conductors compared to the $80 \mu \mathrm{m}$ foil in [29], and the $120 \mu \mathrm{m}$ thread in [28]. In addition, the low surface roughness of copper laminates $(<1 \mu \mathrm{m})$ and higher conductivity than conductive textiles imply lower conductor losses and therefore improved antenna efficiency. Furthermore, the reliability of electronic systems realized using polyimide laminates and encapsulated using glob-top has been extensively studied in [30] showing sustained functionality over more than 1000 bending cycles.

Although the textile substrate in use has previously been characterized at $2.45 \mathrm{GHz}$ in [22], the inhomogeneity of textiles mean that a new two-line characterization [31] of the sample needed to be performed. Two microstrip lines, of approximately $50 \Omega$ impedance were built and measured. the extracted dielectric properties exclude the impedance mismatch losses due to additional reflection at the coax-microstrip interface. The measured insertion loss is $0.90 \mathrm{~dB} / \mathrm{cm}$ and 1.79 $\mathrm{dB} / \mathrm{cm}$ at $28 \mathrm{GHz}$ and $60 \mathrm{GHz}$ respectively, only $0.11 \mathrm{~dB} / \mathrm{cm}$ higher at $60 \mathrm{GHz}$ than reported in [23]. The dielectric properties of the polyimide-woven polyester sandwich substrate utilized is $\epsilon_{r}=1.67$ and $\tan \delta=0.027$.

\section{Antenna Simulation And Measurement}

The 3D model of the antenna has been simulated using fullwave electromagnetic simulation in CST Microwave Studio. The $1.85 \mathrm{~mm}$ connector used to measure the fabricated antenna has been included in the antenna's model to observe its effect both on the antenna's impedance bandwidth and on the radiation patterns, in addition to obtaining a closer match with the measured results for validating the simulation model. A human skin layer, based on CST's Voxel library has been added to investigate the effect of human proximity on the antenna. As a significant portion mmWaves do not penetrate deeper than skin layers due to absorption [32], only the skin layer has been included in the model. Furthermore, as the antenna utilizes a solid reflector plane, minimal power from the antenna's power would be absorbed by the body. When the antenna is simulated at $4 \mathrm{~mm}$ from the skin in CST Microwave Studio [19], 1\% of the accepted power by the antenna has been absorbed by the skin layer.

The fabricated antenna has been measured using a E8361A 67 GHz PNA Vector Network Analyser (VNA), calibrated using a standard Short, Open, Load, Through (SOLT) electronic

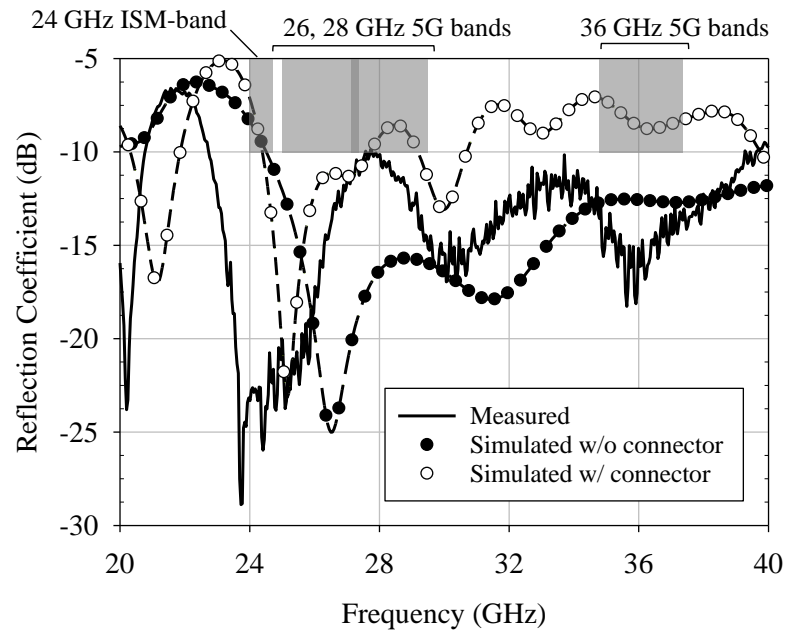

Fig. 6. Simulated (dashed) with (w/) and without (w/o) the connector, and measured (solid) reflection coefficient of the proposed textile mmWave antenna.

calibration kit. The $1.85 \mathrm{~mm}$ connector has been soldered to the bottom ground plane using a uniform solder layer to avoid impedance mismatch at the coax-microstrip transition. Fig. 6 shows the simulated and measured reflection coefficient of the antenna, showing a $S_{11}$ bandwidth covering the full $5 \mathrm{G}$ spectrum up to $40 \mathrm{GHz}$. The deviation in the measured results could be attributed to the connector being of comparable size to the antenna's radiator, and the additional reflection due to standing waves in the test-cable (resulting in the ripples around 24 and $32 \mathrm{GHz}$ ). Nevertheless, the simulation and measurements agree in covering the required $S_{11}$ bandwidth of $24 \mathrm{GHz}$ and $5 \mathrm{G}$ RFEH. Furthermore, the level of agreement at the frequency bands of interest is consistent with similar designs such as the $24 \mathrm{GHz}$ antenna array in [7]. The $\mathrm{S}_{11}$ measurements have been performed with the antenna placed on a human hand and no change has been observed on the measured $S_{11}$, this has also been previously presented in [19] despite the reduced isolation over the proposed reflectorbacked AVA.

For evaluating the far-field properties of the AVA, two designs have been considered: the 25-mm microstrip-fed AVA in Fig. 1 (simulated and measured), and the same antenna with a short 5-mm feed to match the antenna integrated in the final rectenna (simulated). The longer feed sample has been used for model-validation through 3D far-field measurements, to avoid distortion to the measurements due to the connector size.

The 3D polarimetric far-field radiation patterns of the antenna have been measured in an anechoic chamber, at 24 $\mathrm{GHz}$, and simulated using CST's field monitors. A WR-42 horn antenna has been used as a reference for the anechoic chamber measurements (Fig. 5). An extended microstrip feedline of $25 \mathrm{~mm}$ has been included to mitigate distortion from the connector to the radiation patterns [23]. However, the insertion losses in the microstrip line are expected to reduce the measured radiation efficiency of the antenna. The efficiency of the proposed textile antenna has been measured with respect to the horn antenna to be $67 \%$, including the $-1.5 \mathrm{~dB}$ losses 


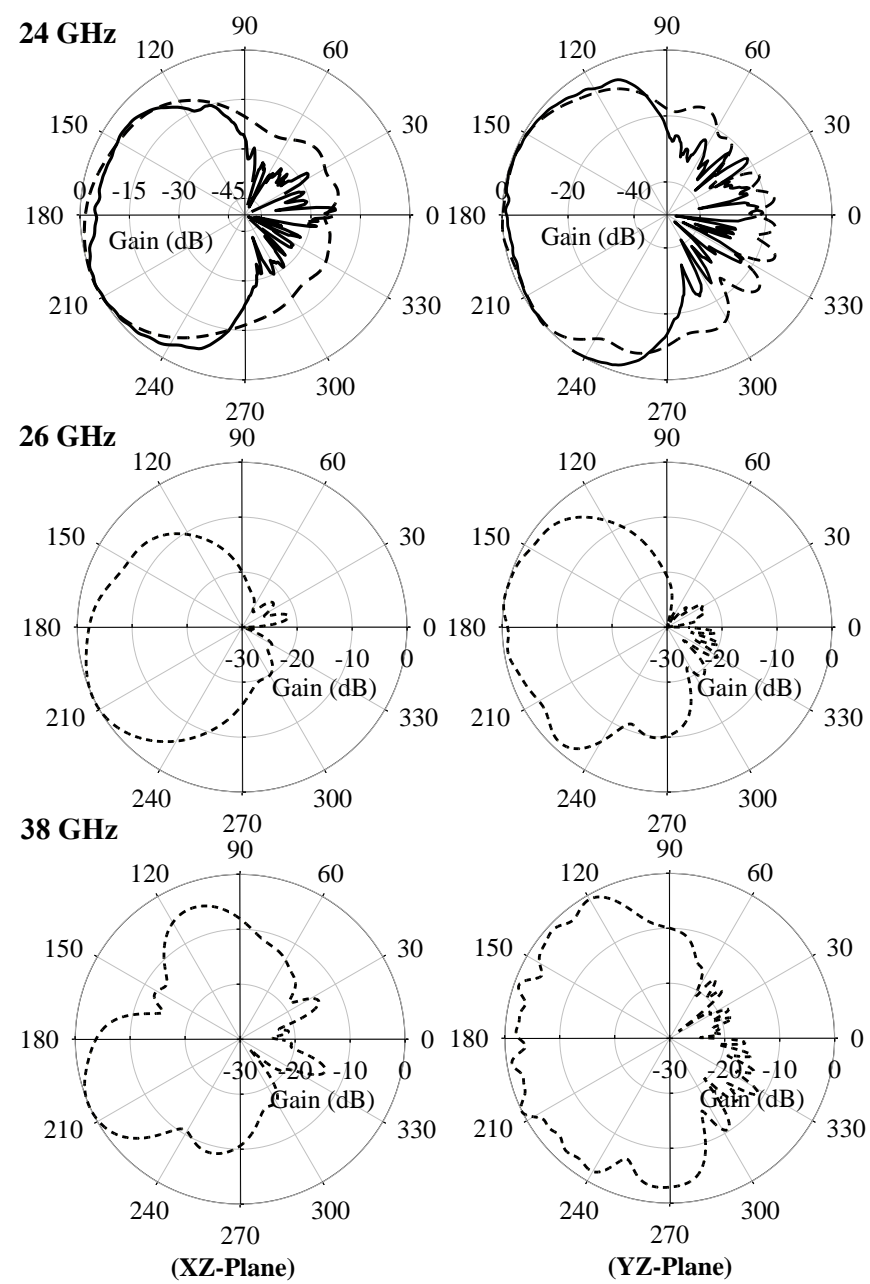

Fig. 7. Normalized simulated (dashed) and measured (solid) gain of the proposed AVA at: (a) 24, (b) 26 and (c) $36 \mathrm{GHz}$, on the XZ and YZ planes of the antenna.

in the prolonged microstrip feed. The normalized gain patterns of the antenna at 24, 28 and $36 \mathrm{GHz}$ are shown in Fig. 7. Both the simulated and measured radiation patterns show over 20 $\mathrm{dB}$ front-to-back ratio, implying minimal interaction between the antenna's broadside radiation and the human body shielded by the reflector. The computed radiation efficiency and gain (validated by the de-embedded measured radiation patterns and efficiency agreement with the simulated results) are shown in Fig. 8. The only discrepancy observed between the simulated and measured radiation properties is the higher back-radiation in the simulated model, due to the smaller size of the reflector in the model to reduce the problem's mesh size. However, in real-life operation and in the measured prototype, the textile reflector could be electrically large minimizing the back-lobe radiation as observed in the measured plots.

From the rectenna's radiation properties shown in Fig. 8 (excluding the simulated insertion losses in the long feedline), the antenna achieves a radiation efficiency of more than $70 \%$ up to $30 \mathrm{GHz}$, peaking at $73.4 \%$ at $26 \mathrm{GHz}$. The decay in efficiency at higher frequencies is due to the increase in the dielectric losses in the substrate. The proposed antenna with the reflector plane achieves slightly lower efficiency

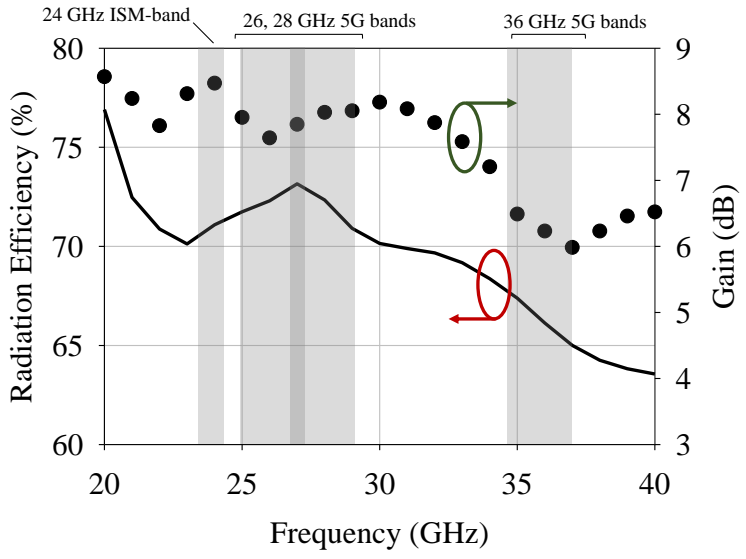

Fig. 8. De-embedded radiation efficiency and gain of the proposed AVA, on-body, with a $5 \mathrm{~mm}$ microstrip feed matching the rectifier's feed length.

TABLE I

COMPARISON OF THE PROPOSED ANTENNA MEASURED PROPERTIES WITH STATE-OF-THE-ART PRINTED AND TEXTILE ANTENNAS

\begin{tabular}{|c|c|c|c|c|}
\hline & This work & 2019 [33] & 2013 [23] & 2012 [34] \\
\hline $\mathrm{BW}(\mathrm{GHz})$ & $\begin{array}{l}23-40 \\
(54.0 \%)\end{array}$ & $\begin{array}{l}26-40 \\
(42.4 \%)\end{array}$ & $\begin{array}{l}55-65 \\
(16.7 \%)\end{array}$ & $\begin{array}{l}57-64 \\
(11.6 \%)\end{array}$ \\
\hline Antenna & AVA & $\begin{array}{l}\mathrm{CPW} \\
\text { monopole }\end{array}$ & $2 \times 2$ patch & Yagi-Uda \\
\hline Substrate & $\begin{array}{l}\text { Textile } \\
(\tan \delta=0.027)\end{array}$ & $\begin{array}{l}\mathrm{LCP} \\
(\tan \delta=0.002)\end{array}$ & $\begin{array}{l}\text { Textile } \\
(\tan \delta=0.02)\end{array}$ & $\begin{array}{l}\text { Textile } \\
(\tan \delta=0.016)\end{array}$ \\
\hline Gain $(\mathrm{dB})$ & $\begin{array}{l}7.41^{*}, \\
8.47 \dagger\end{array}$ & 8.76 & 7.8 & 11.9 \\
\hline $\begin{array}{l}\text { Radiation } \\
\text { pattern }\end{array}$ & $\begin{array}{l}\text { Broadside } \\
\text { single- } \\
\text { beam }\end{array}$ & $\begin{array}{l}\text { Broadside } \\
\text { two-beams }\end{array}$ & $\begin{array}{l}\text { Broadside } \\
\text { single- } \\
\text { beam }\end{array}$ & End-fire \\
\hline Efficiency & $\begin{array}{l}67 \% * \\
73 \% \dagger\end{array}$ & $\begin{array}{l}90 \% \text { (simu- } \\
\text { lated) }\end{array}$ & $41 \%$ & $48 \%$ \\
\hline $\begin{array}{l}\text { Electric } \\
\text { dimensions } \\
\left(\lambda_{0}\right)\end{array}$ & $\begin{array}{ll}3.0 \lambda_{0} & \times \\
0.94 \lambda_{0} & \end{array}$ & $\begin{array}{l}1.12 \lambda_{0} \times \\
1.02 \lambda_{0} \ddagger\end{array}$ & $\begin{array}{ll}4.26 \lambda_{0} & \times \\
1.03 \lambda_{0} & \end{array}$ & $5.2 \lambda_{0} \times 1.6 \lambda_{0}$ \\
\hline
\end{tabular}

$\ddagger$ Small length due to the miniaturized CPW feed and connector's ground.

than the previously simulated $77 \%$ in [19]. However, the solid reflector results in more stable radiation properties over the full bandwidth, as observed in Fig. 8, in addition to guaranteeing that the achieved efficiency does not degrade in human proximity.

Table I compares the performance of the proposed AVA against reported textile-based and additively manufactured low-cost mmWave antennas. It can be seen that the proposed antenna achieves the highest radiation efficiency compared to the reported antennas implemented on low-cost substrates. The antenna in [33] is able to achieve efficiencies higher than $90 \%$ due to the low loss substrate and its CPW geometry. To add, the smaller length of [33] does not include a sufficiently large ground plane due to the coax connector acting as a ground plane for the antenna in simulation and measurement.

\section{Textile MmWaVe RectifieR}

\section{A. Diode Selection and Modeling}

The main criteria when choosing a diode at mmWave bands is the high cut-off frequency of the diode, otherwise the diode will not act as a rectifying element and the high-frequency 
signal will be able to propagate to the output through the diode and package parasitics. While the cut-off frequency is the frequency when the real and imaginary components of the diode impedance are equal, the diode's Power Conversion Efficiency (PCE) will be adversely reduced at frequencies approaching $f_{\text {cut-off }}$. Moreover, a significant portion of the energy at the output will always exist at the $1^{\text {st }}$ and $2^{\text {nd }}$ harmonics and not at DC [8], implying that proper harmonic termination is implemented at the output [7], [12], [14]. The cut-off frequency is defined in this work as the frequency where the resistance and the reactance of the diode are equal, and is given by

$$
f_{\text {cut-off }}=\frac{1}{2 \pi R_{S} C_{T}},
$$

where $C_{T}$ is the diode's total capacitance and $R_{S}$ is the series resistance [35], [36]. While in [36] only the junction capacitance is considered due to the devices being measured on-wafer, when the diodes are packaged the total (junction+packaging) capacitance needs to be considered.

Two diodes have been considered for the rectifying element: a Silicon Skyworks SMS7630 and a Gallium Arsenide (GaAs) Macom MA4E-1319, recommended by the manufacturers for detector applications above 26 and $80 \mathrm{GHz}$, respectively. Extensive analytical and experimental analysis of the PCE of different rectifiers (including the SMS7630 and MA4E) is presented in [37]. The purpose of the input impedance simulation is to highlight the variation in the performance of the diodes when the packaging parasitics are considered, which is particularly important in a flexible textile-based system. The MA4E has previously been simulated demonstrating the highest PCE compared to commercial $\mathrm{Si}$ diodes at $24 \mathrm{GHz}$ [38]. The diodes have been simulated, in a single series topology, in ADS using Harmonic Balance simulation. Both $50 \Omega$ source and load impedance were used to match the measurement setup of the two-port s-parameters.

To maximize the simulation accuracy between 20 and 30 $\mathrm{GHz}$, the diode models were based on the datasheet SPICE parameters, and optimized models using the available measured s-parameters (SMS7630) and the IV-curves (MA4E) [12]. Fig. 9 shows the simulated input impedance of the SMS7630 and the MA4E, based on the $0 \mathrm{dBm}$ s-parameters and the IV-curve, respectively, when terminated with an ideal $50 \Omega$ load. The Smith chart in Fig. 9 shows the cut-off region of the diodes' impedance (striped shading) and the rectification region (solidgreen shading). The diode parameters used in the non-linear simulations in this work are $C_{j}=0.02 \mathrm{pF} R_{S}=9 \Omega$ for the junction capacitance and series resistance, and $C_{P}=0.035 \mathrm{pF}$ $L_{P}=0.3 \mathrm{nH}$ for the packaging capacitance and inductance.

It can be observed that although the SMS7630 junction (datasheet parameters) is capable of rectification up to $24 \mathrm{GHz}$ (impedance falling in the solid-green shaded region in Fig. 9). However, when the packaged diode's parasitics are taken into consideration, the diode acts as a short-circuit allowing the $1^{\text {st }}$ order harmonics to propagate to the output. The MA4E1319 on the other hand is usable up to $40 \mathrm{GHz}$ (based on the LargeSignal S-Parameters LSSP-simulation using the parameters from [12]), yet the packaging parasitics show significant

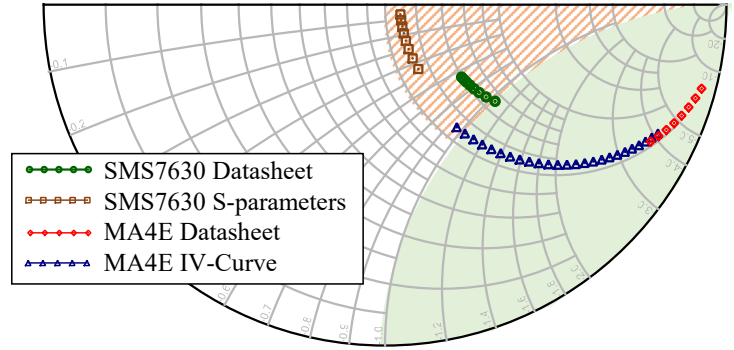

Fig. 9. Simulated (LSSP) input impedance of the SMS7630 and MA4E diodes from 20 to $40 \mathrm{GHz}$, using diode parameters from the datasheet as well as the optimized models based on experimental characterization.

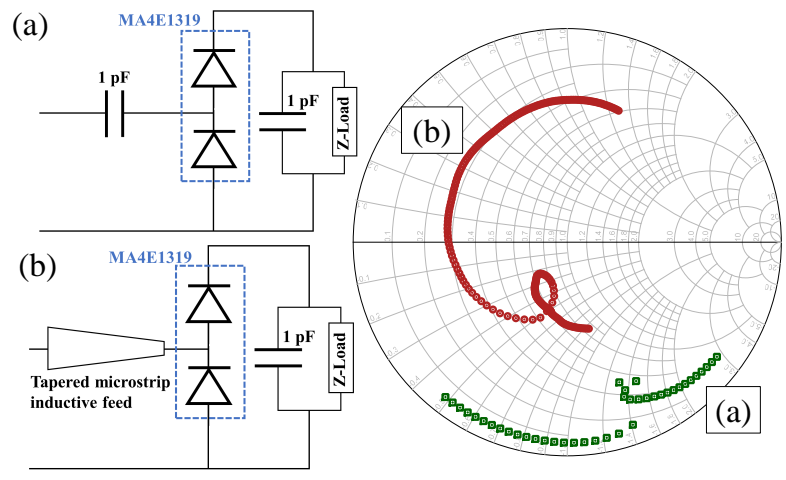

Fig. 10. Schematic of the voltage doubler and Smith charts showing the impedance matching approach, for a $10 \mathrm{k} \Omega$ load, of: (a) conventional voltage doubler, (b) rectifier with the tapered matching line and distributed microstrip input capacitance.

discrepancy with the datasheet model of the junction which can be used through $100 \mathrm{GHz}$. The discrepancies could be attributed to the diode's mounting imperfections while the manufacturer's parameters are measured on-wafer.

\section{B. Rectifier Design}

The MA4E-1319 has been chosen as the rectifying element in this work. With two diode's integrated in the same die, it is expected that the external parasitics will be lower improving the cut-off frequency of the diode. The voltage doubler's impedance, seen on the Smith chart in Fig. 10$\mathrm{a}$, is predominantly capacitive. Therefore, a high-impedance inductive matching network is required. Moreover, the $1 \mathrm{pF}$ input capacitance can be achieved using the microstrip feed's own distributed capacitance. A tapered microstrip line could be used to match the impedance of the rectifier as in Fig. 10-b, stepping-up the impedance of the rectifier to match the $50 \Omega$ source, as well as conjugating the capacitive diode impedance using the microstrip line's own inductance.

A key challenge in broadband impedance matching is the trade-off between bandwidth and Quality factor, where a sufficiently low $S_{11}$ may not be achievable over a wide range of frequencies. The theoretical limit on the minimum achievable reflection coefficient of a load is given by the Bode-Fano return-loss limit,

$$
\begin{gathered}
\left|\Gamma_{m}\right| \geq e^{\frac{-1}{2 B R C}} \\
S_{11} \geq 20 \log \left(\Gamma_{m}\right),
\end{gathered}
$$


a function of the bandwidth $B$, and the equivalent $\mathrm{RC}$ circuit of the load. The load in this case is the voltage doubler and the DC load.

Harmonic balance simulation was used to extract the rectifier's impedance (Fig. 10-a), the bandwidth $B$ has been set to $4 \mathrm{GHz}(24-28 \mathrm{GHz})$. The equivalent $\mathrm{RC}$ circuit of the rectifier in Fig. 10-a has been extracted using optimization of an RC load with tunable resistance and capacitance, to match the simulated LSSP; the extracted values are $\mathrm{R}=904 \Omega$ and $\mathrm{C}=0.161 \mathrm{pF}$. The calculated minimum achievable Fano $\mathrm{S}_{11}$ is $-7.5 \mathrm{~dB}\left(\Gamma_{m} \geq 0.42\right)$. While in [39] the increase in the number of diodes has been studied in order to increase the return loss of a rectifier through reducing $R$, this approach is not possible at mmWave frequencies due to the higher losses incurred in the additional rectifier stages. In [40], the impact of the diode's model on the maximum achievable bandwidth and return loss is studied, showing that a diode with lower parasitics, such as the VDI ZBD, can achieve over $10 \times$ higher bandwidth compared to the SMS7630. However, the high cost of the W-band VDI ZBD diode makes it impractical for low-cost wearable IoT applications. Therefore the MA4E is selected.

In order to step up the input impedance of the $50 \Omega$ source and achieve an inductive feed-line, the microstrip line feeding the diode is tapered to gradually increase the inductance of the source. A tapered line is also used at the ground connection of the voltage doubler, the length of the tapered lines has been optimized in simulation to achieve an impedance match between the $50 \Omega$ antenna and the rectifier at the frequencies of interest. While an L-matching network could be used to achieve a high Q-factor matching, improving the PCE at low $\mathrm{P}_{R F}$ levels as in [7] and [41], a wide bandwidth rectifier is prioritized in order to demonstrate the feasibility of harvesting from a broad bandwidth to meet $5 \mathrm{G}$ RFEH requirements.

In order to prevent the high-frequency component of the input from propagating to the output, significantly reducing the net DC output, radial stubs $(\lambda / 4$ at 26 and $52 \mathrm{GHz})$ are utilized for harmonic rejection at the output. The DC test pads included at the output, to allow measuring the voltage across the load, were found to not have any effect on the rectifier's performance in simulation. Fig. 11 shows the layout of the rectifier, and the modified antenna ground plane to accommodate the rectifier. As the ground via is for the DC return current, the impedance matching of the vias is not critical. Furthermore, different thicknesses, resistances, and inductances of the vias have been studied in ADS and were found to have minimal impact on the rectifier's performance.

In terms of textile coverage and breathability, the proposed rectenna occupies a small area of $16 \times 33 \mathrm{~mm}^{2}$. Thus, should the rectenna be used in a large-area array configuration, a $\lambda_{0} / 2$ $(6.25 \mathrm{~mm}$ at $24 \mathrm{GHz})$ would imply a coverage of less than $60 \%$ of the total area of the garment. Furthermore, [30] has shown scalable integration of polyimide-circuit filaments in standard textile weaves for improved integration, user-comfort and reliability.

\section{Rectifier Simulation and Measurement}

The return loss of the $1.85-\mathrm{mm}$ connector-fed textile rectifier prototype has been measured using a VNA. Due to additional

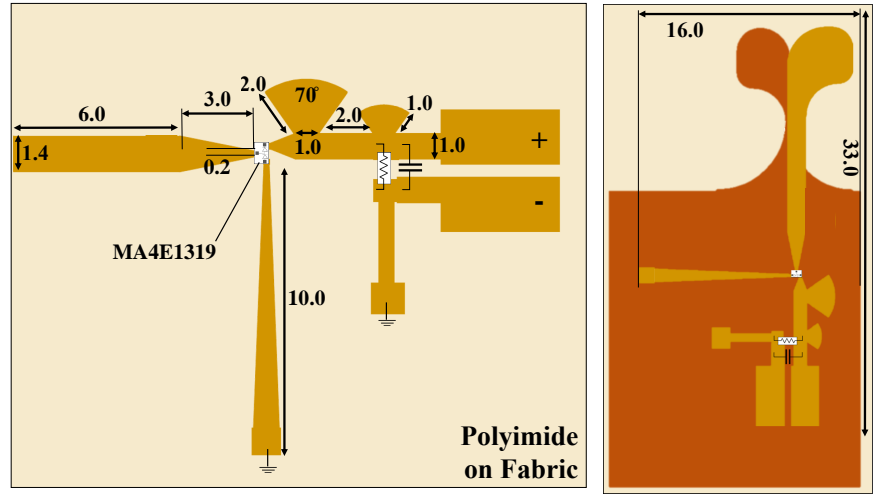

Fig. 11. The dimensions, in $\mathrm{mm}$, of the proposed textile-based $5 \mathrm{G}$ rectifier (left) and the integrated rectenna layout (right).

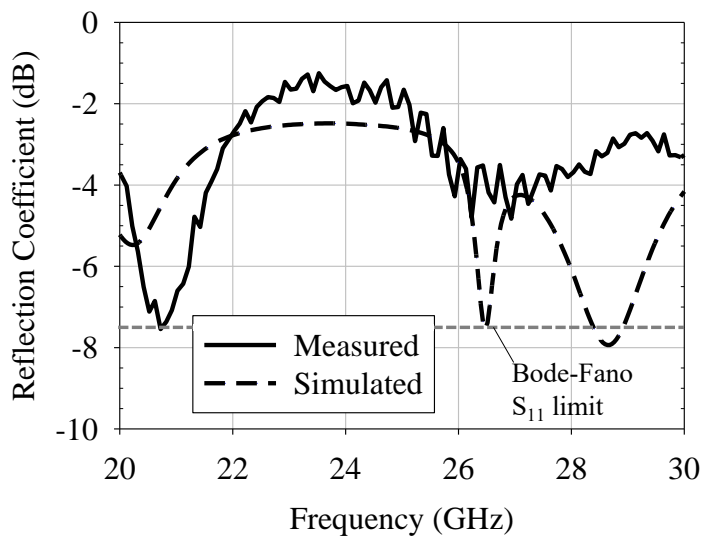

Fig. 12. Measured and simulated reflection coefficient of the rectifier at -5 $\mathrm{dBm}$.

incurred cable losses at $28 \mathrm{GHz}$ and the VNA's power limit, the maximum power deliverable by the VNA is $-5 \mathrm{dBm}$. Fig. 12 shows the simulated and measured $S_{11}$ of the rectifier. The connector has been modeled as a tapered microstrip line of 5 mm length. While the observed reflection is higher than $3 \mathrm{~dB}$, this is attributed to the wide variation in the input impedance of the rectifier due to its large electric length, impeding a sufficiently low $\mathrm{S}_{11}$, in addition to the Bode-Fano theoretical limit on the minimum reflection calculated to be above -7.45 $\mathrm{dB}$.

In order to measure the PCE of the rectifier, the DC voltage across the load has been measured using an oscilloscope. A Wiltron 68069B Continuous Wave (CW) signal generator (providing up to $13 \mathrm{dBm}$ ) has been used to provide sufficient power to investigate the performance of the connectorized rectifier. A Keysight N8485A $33 \mathrm{GHz}$ power meter has been used for power-calibration and for measuring the net power available at the rectifier's input after factoring in cabling and unleveled output losses, reducing the uncertainty in the mmWave power level to $\pm 0.1 \mathrm{~dB}$. Due to the low thickness of the substrate compared to a standard $1.6 \mathrm{~mm}$ board connector, the coax interface introduces additional $0.1 \mathrm{nH}$ inductance (modeled in CST) reducing the rectifier's impedance matching. The power has been swept from $0 \mathrm{dBm}$ to the setup's maximum output of $13 \mathrm{dBm}$. Fig. 13 shows the measured DC output, across a 


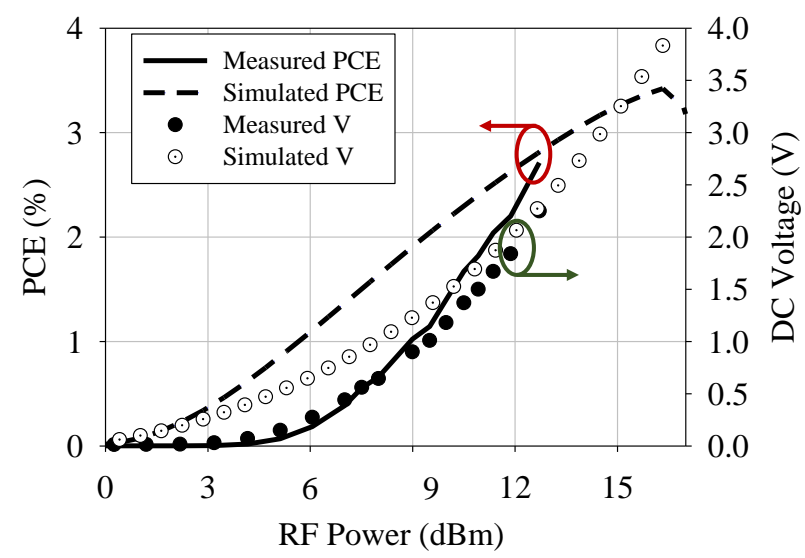

Fig. 13. Measured and simulated PCE of the rectifier and the DC voltage across a $10 \mathrm{k} \Omega$ load at $24 \mathrm{GHz}$ with a coax connector.

\section{$10 \mathrm{k} \Omega$ resistor, at $24 \mathrm{GHz}$.}

Above $10 \mathrm{dBm}$ of input power, it can been seen that the simulated output voltage matches, within $10 \%$, the measured values. The agreement between simulation and measurement shown in Fig. 13 at $10 \mathrm{dBm}$ is higher than that in [7] and [42], where approximately $25 \%$ discrepancy has been reported in the measured voltage output compared to simulation, despite them being implemented on rigid low-loss substrates. This validates the proposed rectifier's performance and design approach for flexible lossy textile substrates. The discrepancy between the simulation and measurement at lower power levels can be attributed to:

1) Inaccuracy in modeling the connector in the Harmonic Balance simulation altering the rectifier's input impedance resulting in additional reflection.

2) Inaccuracy of the MA4E junction and packaging resistance and capacitance at low mmWave power levels reducing the PCE.

3) The imperfect connection between the diodes' pads and the microstrip lines resulting in additional series resistance.

When considering the theoretical limit on the received power, the PCE can be calculated using (4). The DC power is referenced to the theoretical maximum accepted power after factoring out Fano's reflection limit using

$$
P C E_{\text {Fano }}=\frac{P_{D C}}{\left(1-\left|\Gamma_{m}\right|^{2}\right) P_{R F}},
$$

instead of the power available from the generator.

The maximum $\mathrm{PCE}_{F a n o}$ achieved by the rectifier at 24 $\mathrm{GHz}$ is $14 \%$ at $12.7 \mathrm{dBm}$, with a net DC output of $0.5 \mathrm{~mW}$, sufficient for low-power nodes and transceivers in [16]-[18].

The output of the rectifier has been investigated at different load impedances. Fig. 14 shows the PCE of the rectifier at $24 \mathrm{GHz}$ for different load impedances at $10 \mathrm{dBm}$. The higher optimal load impedance of the proposed rectifier compared to the reported mmWave rectifiers (Table II), due to the voltage doubler, configuration results in the improved $1-\mathrm{V}$ sensitivity.

In order to evaluate the rectifier's capability of harvesting energy across a broad bandwidth for $5 \mathrm{G}$ applications, the

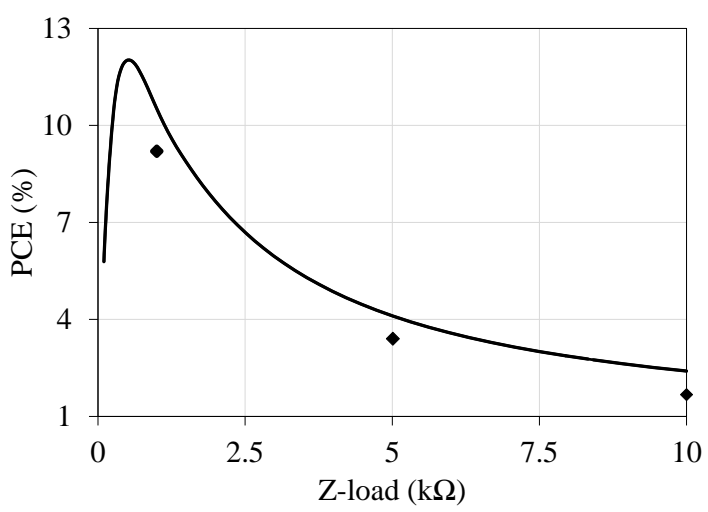

Fig. 14. Simulated (solid line) and measured (discrete points) PCE for variable load impedance at $10 \mathrm{dBm}$.

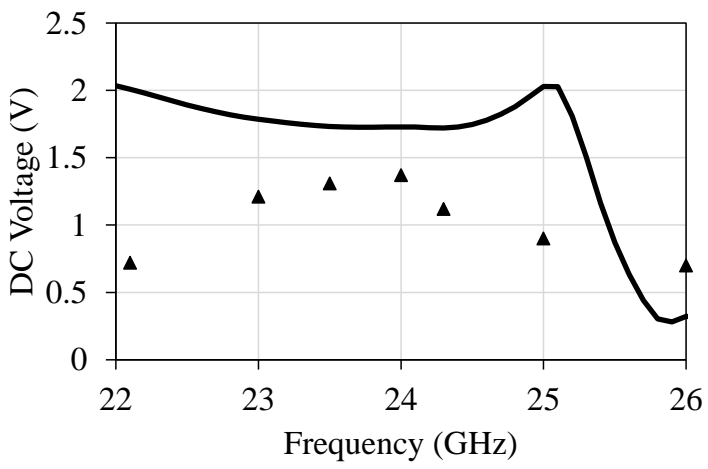

Fig. 15. Simulated (solid line) and measured (discrete points) DC voltage output of the connector-fed textile rectifier at $10 \mathrm{dBm}$ with a $10 \mathrm{k} \Omega$ load.

frequency of the $\mathrm{CW}$ single-tone input to the rectifier has been varied. The load impedance of the broadband rectifier has been set to $10 \mathrm{k} \Omega$, despite the reduced PCE, for improved voltage sensitivity. Fig. 15 shows the output voltage of the rectifier from 22 to $26 \mathrm{GHz}$.

While the measured output voltage at $24 \mathrm{GHz}$ approaches the simulated value, the rectifier's DC output is cut-off at frequencies higher than $24.5 \mathrm{GHz}$ even though its input impedance matching is expected to be maintained based on the small-signal $\mathrm{S}_{11}$ in Fig. 12. This is attributed to the coaxmicrostrip connector interface resulting in lower output voltage at frequencies above $24 \mathrm{GHz}$. Therefore, the broadband wireless testing in the next section has been performed to overcome the additional measurement uncertainties introduced by the coax connector.

\section{Rectenna Wireless Testing and Evaluation}

\section{A. Rectenna Experimental Testing}

The integrated rectenna has been tested using the CW generator, connected to a Keysight 83020A Power Amplifiers (PA), capable of producing up to $27 \mathrm{dBm}$ at $24.5 \mathrm{GHz}$, and a standard $20 \mathrm{dBi}$ horn antenna. To avoid the PA's gain compression, an additional attenuator was introduced between the $\mathrm{CW}$ and the PA to minimize the unleveling of the output and suppress the gain compression effect. Finally, due to the non-linear behavior of the PA and the $\mathrm{CW}$ generator at higher power levels, a calibrated RF power meter has been connected 


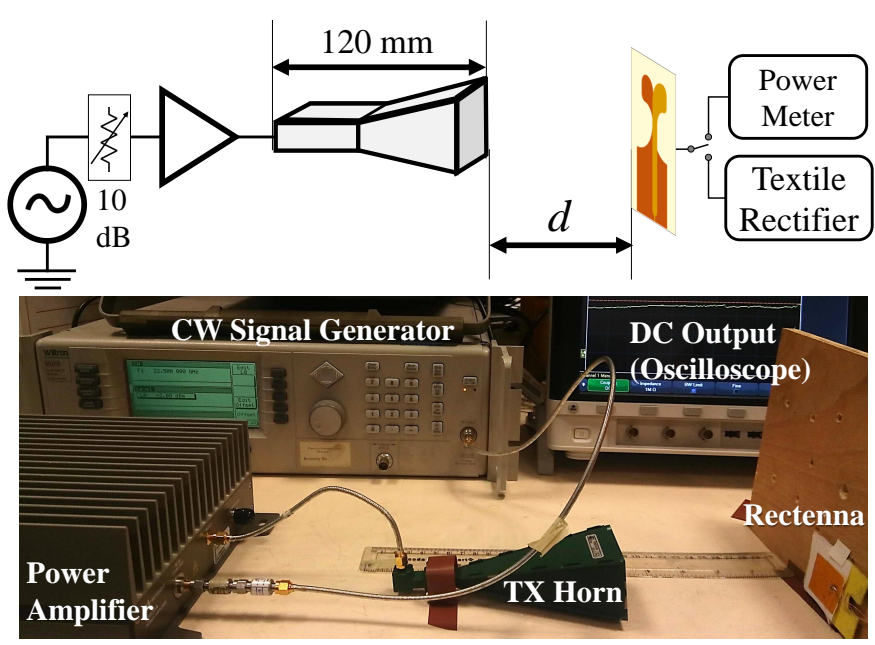

Fig. 16. The wireless test setup schematic and photograph of the textile rectenna.

to the connectorized textile antenna to estimate the power levels at the receiver. The power sensor is rated to a $<1.26$ standing wave ratio (SWR) up to $26.5 \mathrm{GHz}$, normalized to $50 \Omega$. Therefore, the textile AVA is matched to the power sensor ensuring accurate incident power level measurements. The mmWave power levels reported in this section are based on the power calibration using the textile AVA connected to the power meter. Fig. 16 shows the rectenna test setup. The 10 $\mathrm{cm}$ maximum separation between the horn's radiating aperture and the rectenna, shown in Fig. 16, represents $8 \times \lambda$ at $24 \mathrm{GHz}$. This is consistent with the separation reported in the wireless testing of the $17 \mathrm{GHz}$ rectenna in [42] with $8.8 \lambda$ separation between the horn transmitting antenna and the rectenna.

The distance $d$ has been varied to vary the power level at the rectenna. The distance between the horn's radiating aperture and the coax-waveguide transition is $120 \mathrm{~mm}$. Therefore, the total separation between the rectenna and the $\mathrm{CW}$ signal from the PA is $d+120 \mathrm{~mm}$. While the power is typically proportional to $1 / d^{2}$, due to the measurements being carried with $d$ being comparable to the horn's dimensions, the far-field gain and path loss models are not accurate. The input power to the PA in this setup has been kept below $-17 \mathrm{dBm}(-5 \mathrm{dBm}$ signal generator input, $-10 \mathrm{~dB}$ attenuator and $-2 \mathrm{~dB}$ cable losses). The power level is below the PA's saturation region to minimize its non-linearity and enhance repeatability. Fig. 17 shows the measured DC output of the rectenna at varying separation, as well as the measured incident mmWave power, at $24 \mathrm{GHz}$, using the power meter. The distance sweep shows improved voltage-sensitivity over the mmWave rectenna in [41], tested using a $42 \mathrm{dBm}$ Equivalent Isotropically Radiated Power (EIRP) with a $25 \mathrm{~dB}$ horn, by more than $1-\mathrm{V}$ at $20-\mathrm{mm}$ separation from the horn's radiating aperture. $\mathrm{CW}$ excitations have been previously utilized to test the performance of broadband rectifiers in [39], [42].

To evaluate the rectenna's performance at higher power levels, the $\mathrm{CW}$ signal generator output has been increased until a $20 \mathrm{dBm}$ is observed on the wireless power meter. At $24 \mathrm{GHz}$, from an incident power of $20 \mathrm{dBm}$, the rectenna achieves more

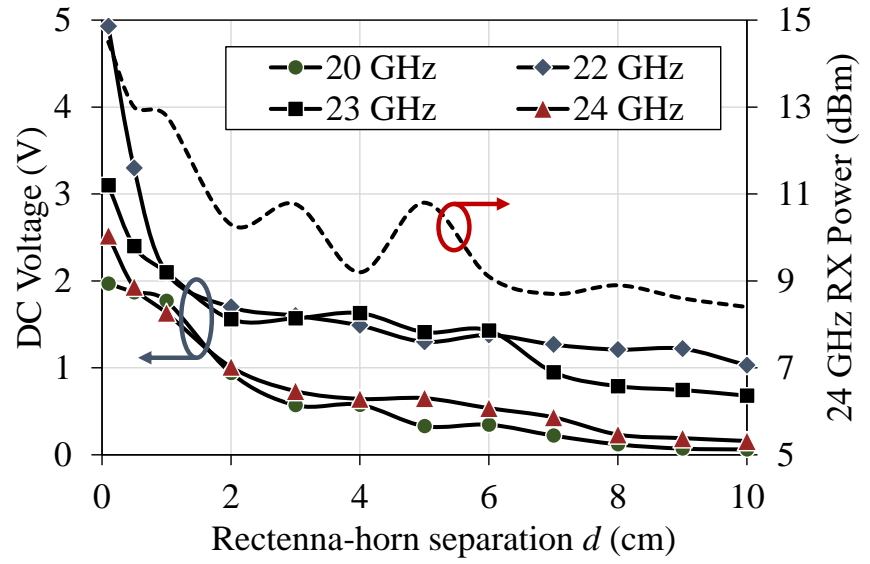

Fig. 17. Measured DC output of the rectenna at variable separation from the horn antenna.

than $6.5 \mathrm{~V}$ with a $10 \mathrm{k} \Omega$ load. This is the highest reported output voltage of a low-power rectenna operating between 20 and $30 \mathrm{GHz}$. The measured DC output of the $10 \mathrm{k} \Omega$-loaded rectenna is $6.5,5.7,4.6$ and 4.2 volts from $20,19,18$, and 16 $\mathrm{dBm}$, respectively. These results have been measured using the test setup in Fig. 16 with the power levels based on the measurement using the power meter.

In order to demonstrate the rectenna's capability of broadband harvesting across the full mmWave 5G spectrum. A broadband frequency sweep has been performed using the wireless setup in Fig. 16. The rectenna has been placed at $d=1 \mathrm{~cm}$ from the horn antenna. To estimate the mmWave power level at the input, the calibrated power meter has been connected to the $50 \Omega$ textile AVA and positioned in the same place as the rectenna.

As the AVA is in the horn's radiative near-field region, the test setup (horn+AVA) has been simulated in CST Microwave Studio to quantify the $\mathrm{S}_{21}$ for additional validation. At 24.5 $\mathrm{GHz}$, the simulated $\mathrm{S}_{21}+\mathrm{PA}^{\prime}$ soutput shows a $\pm 1 \mathrm{~dB}$ agreement with the $12 \mathrm{dBm}$ measured input power using the power sensor. Details of the test setup model are included in the supplementary material. The power from the $\mathrm{CW}$ has been configured at individual measurement points, to overcome the variation in the path loss and the antennas' coupling, to ensure approximately $12 \mathrm{dBm}$ of power is available at the rectenna. Through this power calibration, the gain-compression and PA's non-linearities could be overcome.

As the measured power by the detector is absorbed by its $50 \Omega$ port termination, a different power level will be accepted by the textile rectifier. However, as the textile AVA is matched to a $50 \Omega$ characteristic impedance across its usable bandwidth to simplify measurements, the rectifier's input power (the AVA's microstrip feed) will match that measured by the power sensor. Any variations in the power accepted by the diode will be a function of the impedance matching of the rectifier. While the power evaluation could be performed using a standard gain reference horn antenna, the non-planar geometry of the horn antenna complicates the calculation of the path losses and subsequently the positioning of the rectenna when performing the DC measurements. To explain, as the distance between 
TABLE II

COMPARISON OF THE PROPOSED TEXTILE RECTENNA WITH STATE-OF-THE-ART MMWAVE ENERGY HARVESTERS ABOVE 20 GHZ.

\begin{tabular}{|c|c|c|c|c|c|}
\hline & This Work & TCAS 2014 [7] & AWPL 2014 [41] & WPTC 2017 [12] & IMS 2019 [14] \\
\hline Antenna Design & $\begin{array}{l}\text { Broadband AVA (sin- } \\
\text { gle element) }\end{array}$ & $\begin{array}{l}\text { Cavity-backed } \\
2 \times 2 \text { patch array }\end{array}$ & $4 \times 4$ patch array & $2 \times 2$ patch array & $2 \times 1$ patch array \\
\hline Substrate & $\begin{array}{l}\text { Textile }\left(\epsilon_{r}=1.67,\right. \\
\tan \delta=0.027)\end{array}$ & $\begin{array}{ll}\text { Rogers } & \text { Duroid } \\
(\tan \delta=0.001)\end{array}$ & $\begin{array}{ll}\begin{array}{l}\text { Rogers } \\
(\tan \delta=0.001)\end{array} & \text { Duroid } \\
\end{array}$ & LCP $(\tan \delta=0.003)$ & $\begin{array}{l}\text { 3D printed FLGR02 } \\
\left(\epsilon_{r}=2.83, \tan \delta=0.03\right)\end{array}$ \\
\hline Diode & MA4E-1319 & MA4E-1317 & MA4E-1317 & MA4E-2038 & MA4E-2038 \\
\hline $\begin{array}{ll}\begin{array}{l}\text { Frequency } \\
(\mathrm{GHz})\end{array} & \text { Bands } \\
\end{array}$ & $\begin{array}{l}\text { Antenna: } 22.6-40 \text {, } \\
\text { rectifier: } 20-26.5\end{array}$ & 24 & 35 & 24 & 28 \\
\hline 1-V Sensitivity & $9.5 \mathrm{dBm}(24 \mathrm{GHz})$ & $11.9 \mathrm{dBm}$ & $2.8 \mathrm{~mW}$ & $11 \mathrm{dBm}$ & $12.5 \mathrm{dBm}$ \\
\hline Maximum DC Voltage & $6.5 \mathrm{~V}(20 \mathrm{dBm})$ & $1.2 \mathrm{~V}(13 \mathrm{dBm})$ & $2.2 \mathrm{~V}(8.45 \mathrm{dBm})$ & $2.58 \mathrm{~V}(18 \mathrm{dBm})$ & $2.1 \mathrm{~V}(17 \mathrm{dBm})$ \\
\hline Load $(\mathrm{k} \Omega)$ & $10,0.63$ & 0.16 & 1 & 0.739 & 750 \\
\hline $10 \mathrm{dBm}$ PCE & $12 \%(24 \mathrm{GHz}, 630 \Omega)$ & $24 \%$ & $67 \%(7 \mathrm{~mW})$ & $7.6 \% *$ & $<0.1 \% *$ \\
\hline $\begin{array}{l}\text { Rectenna } 1-\mathrm{V} \text { Band- } \\
\text { width }\end{array}$ & $\begin{array}{l}6.5 \mathrm{GHz} \\
(20-26.5 \mathrm{GHz})\end{array}$ & Single-tone & $1 \mathrm{GHz}$ & Single-tone & Single-tone \\
\hline $\begin{array}{l}\text { Rectenna Half Power } \\
\text { Bandwidth }\end{array}$ & $\begin{array}{l}3.5 \mathrm{GHz}(15 \% \text { frac- } \\
\text { tional bandwidth) }\end{array}$ & Single-tone & Not reported & Single-tone & Single-tone \\
\hline $\begin{array}{l}\text { Electrical Size (rela- } \\
\text { tive to free-space } \lambda \text { ) }\end{array}$ & $2.6 \lambda \times 1.3 \lambda$ & $4.4 \lambda \times 4 \lambda$ & $2.56 \lambda \times 4.9$ & $2.96 \lambda \times 0.89 \lambda$ & Not reported \\
\hline Area $\left(\mathrm{L} \times \mathrm{W}, \mathrm{mm}^{2}\right)$ & $32.6 \times 16$ & $55 \times 50$ & $22 \times 42$ & $37 \times 11.1$ & Not reported \\
\hline
\end{tabular}

*Calculated from the DC voltage curve

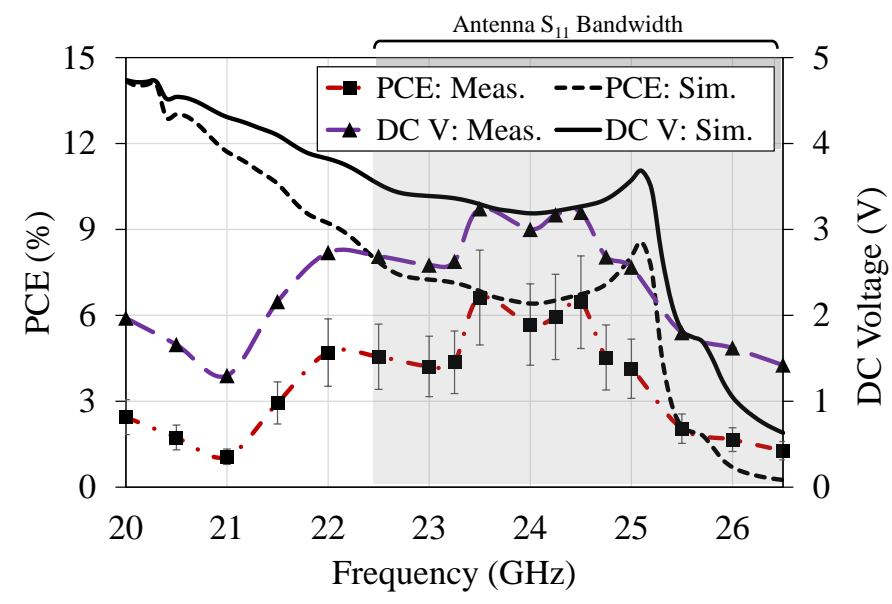

Fig. 18. Measured PCE and DC voltage output of the rectenna from $12 \mathrm{dBm}$ of wireless power, showing more than $1 \mathrm{~V}$ output from 20 to $26.5 \mathrm{GHz}$.

the coax-waveguide transition feeding the horn and the horn's aperture is $120 \mathrm{~mm}$ (larger than $d$ in Fig. 16), identifying a rectenna position where the measured gain of the AVA can be used instead of the horn's would be difficult.

Fig. 18 shows the broadband measured DC voltage the PCE. The error bars reflect the $\pm 1 \mathrm{~dB}$ uncertainty in the incident power level, matching the $1 \mathrm{~dB}$ discrepancy between the path loss simulations and measurements. This demonstrates the rectenna's ability to produce over $1.3 \mathrm{~V}$ DC across the $10 \mathrm{k} \Omega$ load resistor, from $12 \mathrm{dBm}$ of power with PCE varying between 1 and $6.3 \%$ validating its broadband performance. From the measured PCE in Fig. 18, the rectenna achieves a $15 \%$ half-power $(-3 \mathrm{~dB})$ bandwidth from 21.5 to $25 \mathrm{GHz}$.

The simulated values in Fig. 18 were obtained from the ADS model of the rectifier excluding the antenna. However, as the textile AVA is $50 \Omega$ and the wireless power has been measured using the power meter, the results are expected to agree within the $\pm 1 \mathrm{~dB}$ wireless power uncertainty. The main discrepancy between the simulation and measurements shows up below $23 \mathrm{GHz}$. This is attributed to the proposed antenna, which was only designed and characterized for operation from $24 \mathrm{GHz}$. Thus, the PCE in this case is bottle-necked by the antenna. The rectifier design can be integrated with a different antenna should it be used below $24 \mathrm{GHz}$. Above $25.5 \mathrm{GHz}$, the measured output is observed to exceed the simulated output of the rectifier. This could be attributed to the shorter feedlength of the rectifier, when considering the antenna's $50 \Omega$ feed, and the inaccuracies from the resistive and capacitive load mounting. Previously reported mmWave rectennas at 24 $\mathrm{GHz}$ [12], and $94 \mathrm{GHz}$ rectenna [43], have shown a measured DC output higher than the simulated due to overestimating the diode's parasitics.

The measurements were limited to $26.5 \mathrm{GHz}$ due to the PA's and the waveguide horn antenna's maximum frequency. However, as the rectenna achieves more than $1.4 \mathrm{~V}$ at $26.5 \mathrm{GHz}$, it is predicted that the rectenna's cut-off frequency is beyond 27 $\mathrm{GHz}$, demonstrating its capability of harvesting across the full $5 \mathrm{G}$ spectrum. Moreover, the fabricated rectifier's performance above $26 \mathrm{GHz}$ (being significantly better than the simulated due to higher cut-off frequency), could be attributed to overestimation of the parasitic packaging capacitance reproduced from [12], where the utilized dual-diode package results in less parasitics and hence improves the rectifier's usable frequency range. While [42] demonstrates a broadband $1 \mathrm{~V}$ sensitivity from $13 \mathrm{dBm}$ between 14 and $23 \mathrm{GHz}$, this work is the first demonstration of a broadband $1 \mathrm{~V}$ sensitivity above $20 \mathrm{GHz}$, with more than $6.5 \mathrm{GHz}$ of $1.2 \mathrm{~V}$ bandwidth from less than $12 \mathrm{dBm}$ of incident power.

Table II shows a comparison of the proposed rectenna with state-of-the-art mmWave and $5 \mathrm{G}$ rectennas. While it can be seen that the rectennas in [7] and [41] achieve higher efficiency due to the improved single-tone impedance matching, they both have lower load impedance (reducing the voltage sensitivity) in addition to being implemented on dedicated lowloss RF substrates allowing the implementation of large-area distributed elements matching networks. On the other hand, 
despite the high voltage sensitivity achieved by the 3D printed rectenna in [14], implemented on a lossy flexible 3D-printed substrate, the PCE is expected to be very low due to the high load impedance utilized to guarantee a $1 \mathrm{~V}$ DC output. Finally, to the best of the authors' knowledge, this is the first rectenna with a broadband $1 \mathrm{~V}$ sensitivity aimed at broadband mmWave 5G energy harvesting, in addition to being fabricated on textiles with a high-efficiency broadband antenna optimized for operation on-body.

\section{B. Wearable mmWave Powering: Practical Considerations}

Practical deployment of rectennas for radiative mmWave WPT and energy harvesting are dependent on the ability to deliver high power levels to the antenna. The rectenna proposed in this work, as well as in studies [7], [12], [14], require an input power in excess of $9.5 \mathrm{dBm}$ to deliver a $1 \mathrm{~V}$ DC output across a resistive load (as compared in table II). Such a high power level may not be available as ambient radiation to be recycled from cellular communication. Therefore, it is essential to consider the applicability of such rectennas in low-cost mmWave-powered IoT applications.

In the context of harvesting energy in $5 \mathrm{G}$ cellular networks, the $28 \mathrm{GHz}$ EIRP available from a base-station and a mobile device is 75 and $43 \mathrm{dBm} / 100 \mathrm{MHz}$, respectively [44]. Thus, given the rectenna's $7.41 \mathrm{~dB}$ measured realized gain, a mobile phone transmitting at $26 \mathrm{GHz}$ and placed at $10 \mathrm{~cm}$ from the textile rectenna will result $10 \mathrm{dBm}$ received power at the rectenna yielding over $1 \mathrm{~V}$ of DC output. Furthermore, a 1 $\mathrm{V}$ DC output can be achieved with more than $10 \mathrm{~m}$ separation between the rectenna and a mmWave base-station. $10 \mathrm{~m}$ is the size of a typical $10 \mathrm{Gbit} / \mathrm{s}$ 5G urban micro-cell [44]. As a result, the infrastructure (PAs and phased arrays) deployed for high-data rate $5 \mathrm{G}$ communications could support wireless powered networks within the network coverage. Moreover, [4] shows that a wireless-powered network operating at 28 $\mathrm{GHz}$ can achieve over $30 \mathrm{~dB}$ higher power reception for a 400 nodes $/ \mathrm{km}^{2}$ network compared to sub- $6 \mathrm{GHz}$ bands. However, the rectenna in our work has only evaluated the rectenna's performance under $\mathrm{CW}$ excitation, the impact of multi-tone modulated signals on a rectifier's performance have been previously investigated in [45].

High power mmWave power beaming has been shown to improve the power received by a rectenna at a long range $(>600 \mathrm{~m})$ due to the narrow beam of a directional antenna [46]. In [46], the authors study a $7 \mathrm{~kW}$ PA (formed of 8,192 GaN PAs with integrated power combining) which results in an $83 \mathrm{dBm}$ EIRP with a $15 \mathrm{~dB}$ antenna. Although [46] shows that $92 \mathrm{GHz}$ is the frequency where the highest power could be delivered, rectenna implementations beyond $90 \mathrm{GHz}$, such as [43], use diodes with significantly higher costs than the MA4E1319 used in this work. For example, a single Virginia Diodes W-band diode can cost \$15-100, making it unsuitable for low-cost IoT applications. Moreover, to ensure compatibility of the developed antenna and rectifier with future commercial $5 \mathrm{G}$ applications, the choice of frequency in this work is kept below 30 and $40 \mathrm{GHz}$ for the rectifier and the antenna, respectively.

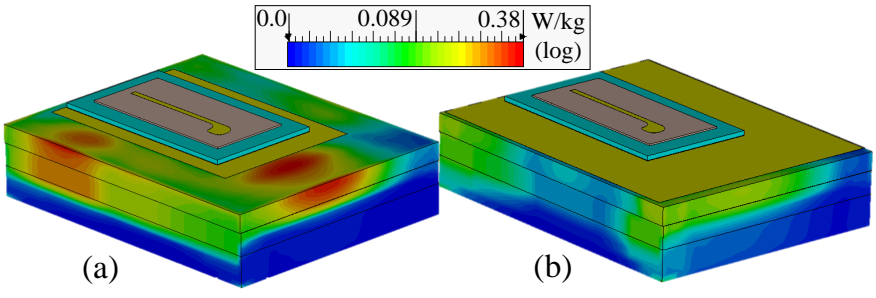

Fig. 19. Simulated SAR of the AVA at $0.1 \mathrm{~mm}$ from the body with: (a) a reflector covering $48 \%$ of the tissue layer, (b) a reflector covering the entire tissue layer.

In wearable operation, mmWave power beaming to on-body devices from an off-body source needs to comply with the safety regulations. In high-power directional WPT applications, multiple control and detection techniques have been proposed to ensure the high-power waves are only directed towards the rectenna to prevent "frying" an unintended target [47]. As the proposed rectenna can be used in large-area energy harvesting arrays, with DC combining as in [48], the full textile reflector backing allows additional shielding of the user.

To further investigate the interaction between the antenna and the human body, the antenna has been simulated in CST Microwave Studio over a human skin model. The uniform 4 mm-tick skin layer is based on the measured tissue dielectric properties in [49] at $28 \mathrm{GHz}$. Above $11 \mathrm{GHz}$, incident waves do not penetrate deeper than $1 \mathrm{~mm}$ while skin is typically 3-4 mm-thick [49]. The skin layer is placed at $0.1 \mathrm{~mm}$ (air gap) from the textile rectenna. A $5 \mathrm{~mm}$ fat and $6.7 \mathrm{~mm}$ muscle layer were added beneath the skin layer. The tissue model is $55 \times 75 \mathrm{~mm}^{2}$. Homogeneous human body models have been used to model the human tissue for both transmitting, [26], and receiving antennas [48].

Given the reciprocal nature of antennas, Specific Absoprtion Rate (SAR) analysis could be used to estimate the absorption at a certain input power level. The proposed rectenna achieves a measured PCE of $4.2 \%$, with a DC output of $6.5 \mathrm{~V}$ across a $10 \mathrm{k} \Omega$ load, from a $20 \mathrm{dBm} 24 \mathrm{GHz}$ input. Thus, it can be assumed that the rectenna could be used to generate power at power levels higher than $20 \mathrm{dBm}$. An input power source of $0.5 \mathrm{~W}(27 \mathrm{dBm})$ is considered for the SAR analysis. Fig. 19-b shows the simulated SAR of the proposed AVA at 28 GHz. For both the compact and full-size reflectors, the peak SAR of 0.38 and $0.092 \mathrm{~W} / \mathrm{kg}$, respectively, confirm that the proposed rectenna could be used safely for wearable highpower mmWave reception while complying with the IEEE C95.1 regulations.

\section{CONCLUSION}

In this paper, a textile-based broadband rectenna for $5 \mathrm{G}$ RF energy harvesting and WPT has been presented based on a novel wearable antenna design and a broadband highsensitivity rectifier. The textile antenna achieves the highest radiation efficiency of reported textile-based mmWave antennas at more than $70 \%$ with a stable gain over $8 \mathrm{~dB}$. The proposed rectenna is capable of generating a $1 \mathrm{~V}$ DC output 
from 21.5 to $25 \mathrm{GHz}$ from power levels as low as $9.5 \mathrm{dBm}$, more than $1.3 \mathrm{~V}$ from 20 to $26.5 \mathrm{GHz}$ from $12 \mathrm{dBm}$ of wireless power, a $15 \%$ half-power fractional bandwidth, and up to $6.5 \mathrm{~V}$ from $20 \mathrm{dBm}$ at $24 \mathrm{GHz}$, the highest voltage compared to reported low-power mmWave rectennas. This work is the first demonstration of a textile-based rectenna operating beyond $20 \mathrm{GHz}$ and the first broadband rectenna beyond for 2026.5 GHz mmWave EH and WPT. This work demonstrates the feasibility of mmWave-powering of unobtrusive sensing and communication implemented on low-cost materials, with the capability of providing a sufficient DC output within the transmission limits of future mmWave $5 \mathrm{G}$ networks, as well as the safety-compliance of the proposed antenna for high-power point-to-point mmWave power transmission.

\section{REFERENCES}

[1] I. A. Hemadeh, K. Satyanarayana, M. El-Hajjar, and L. Hanzo, "Millimeter-Wave Communications: Physical Channel Models, Design Considerations, Antenna Constructions, and Link-Budget," IEEE Communications Surveys \& Tutorials, vol. 20, 2, pp. 870 - 913, 2017.

[2] T. D. P. Perera, D. N. K. Jayakody, S. K. Sharma, S. Chatzinotas, and J. Li, "Simultaneous Wireless Information and Power Transfer (SWIPT): Recent Advances and Future Challenges," IEEE Communication Surveys and Tutorials, vol. 20, 1, pp. $264-302,2018$

[3] A. Costanzo and D. Masotti, "Energizing 5G: Near- and Far-Field Wireless Energy and Data Trantransfer as an Enabling Technology for the 5G IoT," IEEE Microw. Mag., vol. 18, no. 3, pp. 125 - 136, 2017.

[4] T. A. Khan, A. Alkhateeb, and R. W. Heath, "Millimeter Wave Energy Harvesting," IEEE Trans. Wireless Communications, vol. 15, 9, pp. 6048 - 6062, 2016.

[5] L. Wang, K.-K. Wong, R. W. Heath, and J. Yuan, "Wireless powered dense cellular networks: How many small cells do we need?" IEEE Journal on Selected Areas in Communications, vol. 35, no. 9, pp. 2010 - 2024, 2017.

[6] M. Tabesh, N. Dolatsha, A. Arbabian, and A. M. Niknejad, "A PowerHarvesting Pad-Less Millimeter-Sized Radio," IEEE Journal of SolidState Circuits, vol. 50, 4, pp. 962 - 977, 2015.

[7] S. Ladan, A. B. Guntupalli, and K. Wu, "A High-Efficiency $24 \mathrm{GHz}$ Rectenna Development Towards Millimeter-Wave Energy Harvesting and Wireless Power Transmission," IEEE Trans. Circuits And Systems, vol. 61,12 , pp. $3358-3366,2014$.

[8] S. Ladan and K. Wu, "Nonlinear Modeling and Harmonic Recycling of Millimeter-Wave Rectifier Circuit," IEEE Trans. Microw. Theory Techn., vol. 63, 3, pp. $937-944,2015$.

[9] M. Nariman, F. Shirinfar, A. P. Toda, S. Pamarti, A. Rofougaran, and F. D. Flaviis, "A Compact $60-\mathrm{GHz}$ Wireless Power Transfer System," IEEE Trans. Microw. Theory Techn., vol. 64, no. 8, pp. $2664-2677$, 2016.

[10] A. Collado and A. Georgiadis, "24 ghz substrate integrated waveguide (siw) rectenna for energy harvesting and wireless power transmission," in IEEE MTT-S International Microwave Symposium Digest (MTT), 2013.

[11] S. Daskalakis, J. Kimionis, J. Hester, A. Collado, M. M. Tentzeris, and A. Georgiadis, "Inkjet printed 24 ghz rectenna on paper for millimeter wave identification and wireless power transfer applications," in 2017 IEEE MTT-S International Microwave Workshop Series on Advanced Materials and Processes for RF and THz Applications (IMWS-AMP), 2017.

[12] J. Bito, V. Palazzi, J. Hester, R. Bahr, F. Alimenti, P. Mezzanotte, L. Roselli, and M. M. Tentzeris, "Millimeter-wave ink-jet printed RF energy harvester for next generation flexible electronics," in 2017 IEEE Wireless Power Transfer Conference (WPTC), 2017.

[13] M. Wagih, A. S. Weddell, and S. Beeby, "Rectennas for RF Energy Harvesting and Wireless Power Transfer: a Review of Antenna Design," IEEE Antennas and Propagation Magazine, vol. Accepted: In Press, 2019.

[14] T.-H. Lin, S. N. Daskalakis, A. Georgiadis, and M. M. Tentzeris, "Achieving fully autonomous system-on-package designs: An embedded-on-package $5 \mathrm{~g}$ energy harvester within $3 \mathrm{~d}$ printed multilayer flexible packaging structures," in 2019 IEEE MTT-S International Microwave Symposium (IMS), 2019.
[15] A. Eid, J. Hester, and M. M. Tentzeris, "A Scalable High-Gain and Large-Beamwidth mm-Wave Harvesting Approach for 5G-powered IoT," in 2019 IEEE MTT-S International Microwave Symposium (IMS), 2019.

[16] Y. Rajavi, M. Taghivand, K. Aggarwal, A. Ma, and A. S. Y. Poon, "An rf-powered fdd radio for neural microimplants," IEEE Journal of SolidState Circuits, vol. 52, no. 5, pp. 1221 - 1229, 2017.

[17] S. Yang, J. Yin, H. Yi, W.-H. Yu, P.-I. Mak, and R. P. Martins, “A 0.2-V Energy-Harvesting BLE Transmitter With a Micropower Manager Achieving 25\% System Efficiency at 0-dBm Output and 5.2-nW Sleep Power in 28-nm CMOS," IEEE Journal of Solid-State Circuits, vol. 54, no. 5, pp. $1351-1362,2019$.

[18] M. Taghivand, K. Aggarwal, Y. Rajavi, and A. S. Y. Poon, "An Energy Harvesting 2x2 $60 \mathrm{GHz}$ Transceiver With Scalable Data Rate of 38=2450 $\mathrm{Mb} / \mathrm{s}$ for Near-Range Communication," IEEE Journal of Solid State Circuits, vol. 50, 8, pp. 1889 - 1902, 2015.

[19] M. Wagih, A. S. Weddell, and S. Beeby, "Millimeter-Wave Textile Antenna for On-Body RF Energy Harvesting in Future 5G Networks," in 2019 IEEE Wireless Power Transfer Conference (WPTC), 2019.

[20] 5G Spectrum Positions. [Online]. Available: www.gsma.com/spectrum/wp-content/uploads/2019/02/5G-SpectrumPositions-InfoG.pdf

[21] M. Moosazadeh, S. Kharkovsky, J. T. Case, and B. Samali, "Improved Radiation Characteristics of Small Antipodal Vivaldi Antenna for Microwave and Millimeter-Wave Imaging Applications," IEEE Antennas Wireless Propag. Lett., vol. 16, pp. 1961 - 1964, 2017.

[22] S.-E. Adami, P. Proynov, G. S. Hilton, G. Yang, C. Zhang, D. Zhu, Y. Li, S. P. Beeby, I. J. Craddock, and B. H. Stark, "A Flexible 2.45$\mathrm{GHz}$ Power Harvesting Wristband With Net System Output From -24.3 $\mathrm{dBm}$ of RF Power," IEEE Trans. Microw. Theory Techn., vol. 66, 1, pp. 380-395, 2018

[23] N. Chahat, M. Zhadobov, S. A. Muhammad, L. L. Coq, and R. Sauleau, "60-GHz Textile Antenna Array for Body-Centric Communications," IEEE Trans. Antennas Propag., vol. 61, 4, pp. 1816 - 1824, 2013.

[24] B. S. Cook, B. Tehrani, J. R. Cooper, and M. M. Tentzeris, "Multilayer Inkjet Printing of Millimeter-Wave Proximity-Fed Patch Arrays on Flexible Substrates," IEEE Antennas Wireless Propag. Lett., vol. 12, pp. $1351-1354,2013$.

[25] H. R. Khaleel, "Design and Fabrication of Compact Inkjet Printed Antennas for Integration Within Flexible and Wearable Electronics," IEEE Trans. Components, Packaging and Manufacturing Technology, vol. 4, no. 10, pp. 1722 - 1728, 2014.

[26] M. Wagih, Y. Wei, and S. Beeby, "Flexible $2.4 \mathrm{GHz}$ Sensor Node for Body Area Networks with a Compact High-Gain Planar Antenna," IEEE Antennas Wireless Propag. Lett., vol. 17, 12, pp. 49 - 53, 2018.

[27] A. Dierck, H. Rogier, and F. Declercq, "A Wearable Active Antenna for Global Positioning System and Satellite Phone," IEEE Trans. Antennas Propag., vol. 61, no. 2, pp. 532 - 538, 2013.

[28] A. Kiourti, C. Lee, and J. L. Volakis, "Fabrication of Textile Antennas and Circuits With $0.1 \mathrm{~mm}$ Precision," IEEE Antennas Wireless Propag. Lett., vol. 15, pp. $151-153,2016$.

[29] N. Chahat, M. Zhadobov, L. L. Coq, S. I. Alekseev, and R. Sauleau, "Characterization of the Interactions Between a 60-GHz Antenna and the Human Body in an Off-Body Scenario," IEEE Trans. Antennas Propag., vol. 60,12 , pp. $5958-5965,2012$.

[30] A. Komolafe, Y. Torah, Russel ans Wei, H. Nunesmatos, M. Li, D. Hardy, T. Dias, M. Tudor, and S. Beeby, "Integrating flexible filament circuits for etextile applications," Advanced Materials Technologies, vol. 4, no. 7, 2019.

[31] M.-Q. Lee and S. Nam, "An accurate broadband measurement of substrate dielectric constant," IEEE Microwave and Guided Wave Letters, vol. 6,4 , pp. $168-170,1996$.

[32] A. R. Guraliuc, M. Zhadobov, O. D. Sagazan, and R. Sauleau, "Solid Phantom for Body-Centric Propagation Measurements at $60 \mathrm{GHz}$," IEEE Trans. Microw. Theory Techn., vol. 62, 6, pp. 1373 - 1380, 2014.

[33] S. F. Jilani, M. O. Munoz, Q. H. Abbasi, and A. Alomainy, "MillimeterWave Liquid Crystal Polymer Based Conformal Antenna Array for 5G Applications," IEEE Antennas Wireless Propag. Lett., vol. 18, 1, pp. 84, $88,2019$.

[34] N. Chahat, M. Zhadobov, L. L. Coq, and R. Sauleau, "Wearable Endfire Textile Antenna for On-Body Communications at $60 \mathrm{GHz}$," IEEE Antennas Wireless Propag. Lett., vol. 11, pp. 799 - 802, 2012.

[35] J. Semple, D. G. Georgiadou, G. Wyatt-Moon1, G. Gelinck, and T. D. Anthopoulos, "Flexible diodes for radio frequency (RF) electronics: a materials perspective," Semiconductor Science and Technology, vol. 32, 2017. 
[36] M. K. Matters-Kammerer, L. Tripodi, R. van Langevelde, J. Cumana, and R. H. Jansen, "RF Characterization of Schottky Diodes in 65-nm CMOS," IEEE Trans. Electron Devices, vol. 57 no. 5, pp. 1063 - 1068, 2010.

[37] S. Hemour, Y. Zhao, C. H. P. Lorenz, D. Houssameddine, Y. Gui, C.-M. Hu, and K. Wu, "Towards Low-Power High-Efficiency RF and Microwave Energy Harvesting," IEEE Trans. Microw. Theory Techn., vol. 62 no. 4, pp. 965 - 976, 2014.

[38] S. Ladan, S. Hemour, and K. Wu, "Towards Millimeter-Wave HighEfficiency Rectification for Wireless Energy Harvesting," in 2013 IEEE International Wireless Symposium (IWS), 2013.

[39] J. Kimionis, A. Collado, M. M. Tentzeris, and A. Georgiadis, "Octave and Decade Printed UWB Rectifiers Based on Nonuniform Transmission Lines for Energy Harvesting," IEEE Trans. Microw. Theory Techn., vol. 65 , no. 11 , pp. $4326-4334,2017$.

[40] C. H. P. Lorenz, S. Hemour, and K. Wu, "Physical Mechanism and Theoretical Foundation of Ambient RF Power Harvesting Using ZeroBias Diodes," IEEE Trans. Microw. Theory Techn., vol. 64 no. 7, pp. $2146-2158,2016$

[41] A. Mavaddat, S. H. M. Armaki, and A. R. Erfanian, "Millimeter-Wave Energy Harvesting Using 4x4 Microstrip Patch Antenna Array," IEEE Antennas Wireless Propag. Lett., vol. 14, pp. 515 - 518, 2014.

[42] A. Takacs, H. Aubert, S. Fredon, L. Despoisse, and H. Blondeaux, "Microwave Power Harvesting for Satellite Health Monitoring," IEEE Trans. Microw. Theory Techn., vol. 62, 4, pp. 1090-1098, 2014.

[43] S. Hemour, C. H. P. Lorenz, and K. Wu, "Small-footprint wideband $94 \mathrm{GHz}$ rectifier for swarm micro-robotics," in 2015 IEEE MTT-S International Microwave Symposium, 2015.

[44] Y. Huo, X. Dong, and W. Xu, "5g cellular user equipment: From theory to practical hardware design," IEEE Access, vol. 5, 2017.

[45] F. Bolos, J. Blanco, A. Collado, and A. Georgiadis, "RF Energy Harvesting From Multi-Tone and Digitally Modulated Signals," IEEE Trans. Microw. Theory Techn., vol. 64, no. 6, pp. 1918 - 1927, 2016.

[46] H. Kazemi, K. Shinohara, and C. W. Eckhardt, "Millimeter Wave Wireless Power Transmission-Technologies and Applications," in IEEE Wireless Power Transfer Conference, 2019.

[47] R. M. Dickinson, "Power in the sky: Requirements for microwave wireless power beamers for powering high-altitude platforms," IEEE Microw. Mag., vol. 14 no. 2, pp. 36 - 47, 2013.

[48] D. Vital, S. Bhardwaj, and J. L. Volakis, "Textile Based Large Area RF-Power Harvesting System for Wearable Applications," IEEE Trans. Antennas Propag., vol. Early Access, 2019.

[49] D. Ghodgaonkar, O. Gandhi, and M. Iskander, "Complex permittivity of human skin in vivo in the frequency band $26.5-60 \mathrm{GHz}$," in IEEE Antennas and Propagation Society International Symposium, 2000.

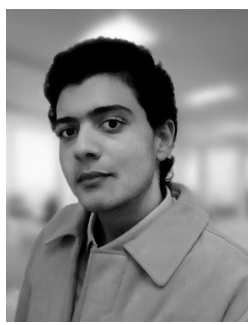

Mahmoud Wagih (GS'18) received his B.Eng (Hons.) from the University of Southampton in 2018, where he is currently pursuing his Ph.D.

In 2017 he worked as a Research Assistant at the University of Southampton, supported by Intel, investigating novel differential transmission lines. In 2018, he was a Hardware Engineering Intern at Arm, UK, and, in 2020, a devices research intern at Arm,. His current research interests lie in RF energy harvesting, wireless power transfer, and mmWave wearable antennas. He has over 20 journal and conference publications in these areas.

Mr. Wagih is a graduate student member of the IEEE. He was the recipient of the Best Undergraduate Project Prize at the University of Southampton, 2018, and was selected for the IEEE MTT-S International Microwave Symposium Project Connect, 2019. He is the recipient of the Best Student Paper Award at the IEEE MTT-S Wireless Power Transfer Conference, 2019, and the Best Oral Paper at PowerMEMS 2019.

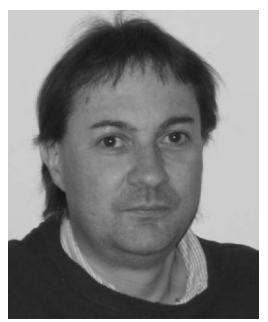

Geoffrey S. Hilton received the B.Sc. degree from the University of Leeds, Leeds, U.K., in 1984, and the Ph.D. degree from the University of Bristol, Bristol, U.K., in 1993, for research on the design and finite-difference time-domain modeling of printed antenna elements.

$\mathrm{He}$ is a Senior Lecturer with the University of Bristol. His current research interests include practical antenna and system design for a variety of communications and radar applications such as ground penetrating radar, performance evaluation of antennas in mobile radio, electrically small elements, active/tuneable elements, and vehicle-mounted conformal antennas.

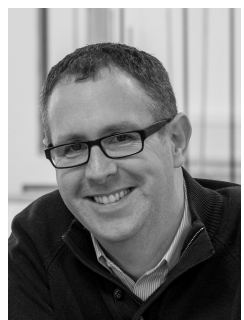

Alex S. Weddell (GS'06-M'10) received the M.Eng. degree (1st class honors) and Ph.D. in electronic engineering from the University of Southampton, U.K., in 2005 and 2010.

His main research focus is in the areas of energy harvesting and energy management for future Internet of Things devices. He has over 14 years experience in design and deployment of energy harvesting systems, and has published around 55 peer-reviewed papers in the area. He is currently a Lecturer in the Center for Internet of Things and Pervasive Systems at the University of Southampton, and is involved with three projects funded by EPSRC, EU Horizon 2020 and Clean Sky 2.

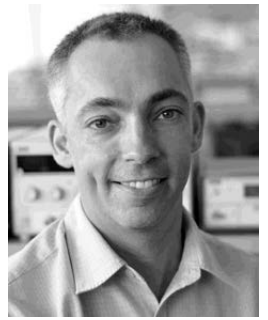

Steve Beeby received the B.Eng. (Hons.) degree in mechanical engineering from the University of Portsmouth, Portsmouth, U.K., in 1992, and the $\mathrm{Ph} . \mathrm{D}$. degree in MEMS resonant sensors from the University of Southampton, Southampton, U.K., in 1998.

$\mathrm{He}$ is currently the Head of the Smart Electronic Materials and Systems Research Group and leads the U.K.'s E-Textiles Network. He is currently leading three U.K. funded research projects and has received over 16 million research funding. $\mathrm{He}$ is a $\mathrm{Co}-$ Founder of Perpetuum Ltd., a University spin-out based upon vibration energy harvesting formed in 2004, Smart Fabric Inks Ltd., and D4 Technology Ltd. He has co-authored/edited four books including Energy Harvesting for Autonomous Systems (Artech House, 2010). He has given 25 invited talks and has over 300 publications and 10 patents. He has an h-Index of 52. His current research interests focus on energy harvesting, e-textiles and the use of energy harvesting in wearable applications.

Dr. Beeby was the recipient of two prestigious EPSRC Research Fellowships to investigate the combination of screen-printed active materials with micromachined structures and textiles for energy harvesting and was also awarded a Personal Chair in 2011. He is currently the Chair of the International Steering Committee for the PowerMEMS Conference series. 